\title{
Relationship between the rs333 Polymorphism in the CC Chemokine Receptor Type Five (CCR5) Gene and Immunological Disorders: Data from a Meta-Analysis
}

\author{
Felipe Rodolfo Pereira da Silva ${ }^{1,4, *}$, Alessandro Luiz Araújo Bentes Leal ${ }^{2}$, Reyce Santos \\ $\mathrm{Koga}^{3}$, Even Herlany Pereira Alves ${ }^{4}$, John Arlley Sousa Pinho de Lira ${ }^{4}$, Humbelina Alves \\ da Silva ${ }^{4}$, Karen Neisman Rodríguez Ayala ${ }^{4}$, Paulo Roberto Carneiro Gomes ${ }^{4}$, Anna \\ Carolina Toledo da Cunha Pereira ${ }^{2}$ and Daniel Fernando Pereira Vasconcelos ${ }^{4}$
}

\author{
${ }^{1}$ Post Graduation Program in Basic and Applied Immunology, Federal University of Amazonas, Manaus, \\ Amazonas, Brazil \\ ${ }^{2}$ Laboratory of Biology of Microorganisms, Parnaiba Delta Federal University (UFDPar), Parnaiba, Piaui, \\ Brazil \\ ${ }^{3}$ Post Graduation Program in Public Health, University of São Paulo, São Paulo, Brazil \\ ${ }^{4}$ Laboratory of Histological Analysis and Preparation (LAPHis), Parnaiba Delta Federal University (UFDPar), \\ Parnaiba, PI, Brazil
}

\begin{abstract}
Introduction: Inflammatory Bowel Disease (IBD), periodontitis and Systemic Lupus Erythematous (SLE) are multifactorial diseases, one of the factors in the course of these diseases is the rs333 polymorphism in the CC chemokine receptor type five (CCR5) gene. However, the results remain contradictory. Therefore, we aimed to perform a meta-analysis evaluating the relation between this polymorphism and the aforementioned conditions.

Material and Methods: A search in the literature was performed in diverse scientific and medical databases for studies published before June 22, 2020. The data were extracted from the studies and the statistical evaluation was performed by the calculations of statistical heterogeneity $\left(I^{2}\right)$, Odds Ratio $(\mathrm{OR})$ with $95 \%$ of Confidence Intervals $(\mathrm{Cl})$ and publication bias. The values of $\mathrm{P}<0.05$ were considered as significant for all calculations.

Results: 19 articles with 21 case/control studies in 4,304 case patients and 3,492 controls were included. The metaanalysis showed a non-significant association among the rs333 polymorphism and IBD (OR $=1.05,95 \% \mathrm{Cl}$ : 0.91-1.20, $\mathrm{P}$ $=0.51$ ), periodontitis $(\mathrm{OR}=0.86,95 \% \mathrm{Cl}: 0.64-1.17, \mathrm{P}=0.34)$ or $\mathrm{SLE}(\mathrm{OR}=1.00,95 \% \mathrm{Cl}: 0.56-1.80, \mathrm{P}=1.00)$ under the allelic model or for any other performed calculation. There were no obvious publication bias in the analyses.
\end{abstract}

Conclusion: In conclusion, this current meta-analysis evidenced the non-significant relation among the rs333 polymorphism and the risk of IBD, periodontitis or SLE. Further studies are required to validate our data.

Keywords: Cytokine, inflammation, autoimmune disease, genetic variation.

\section{INTRODUCTION}

Inflammatory Bowel Disease (IBD), periodontitis and Systemic Lupus Erythematous (SLE) are complex, multifactorial and relevant immunological disorders in the clinical field for the contribution of environmental, genetics and epigenetics factors that contribute to their pathogeneses [1-3].

IBD reached the burden of 5 million globally [4] with considerable prevalence in Netherlands [5] and the value of $4.3 \%$ per year between 2008 and 2018 in Scotland [6]. The main feature of IBD is the longstanding relapsing inflammation that affects various parts of gastrointestinal and it comprises two commonly diseases: the Cronh's Disease (CD) and Ulcerative

*Address correspondence to this author at the Department of Morphology, Federal University of Amazonas, General Rodrigo Octavio Jordão Ramos Avenue, 1200, Coroado, Manaus, Amazonas, CEP: 69067-005, Brazil;

Tel: +5592992873755; E-mail: feliperodolfo.15@hotmail.com
Colitis (UC) [7]. Both clinical types share the same clinical course and outcome; however, it was observed that confined differences to the site of the lesions which $C D$ affects the wall of the gut and UC is represented by lesions in the colon where it extends from the rectum to proximal parts of the large intestine [8].

Periodontal diseases are a group of inflammatory disorders that range the supporting structures around the teeth resulting in possible teeth loss [9]. The aetiology of periodontal diseases is well hallmarked by the dysbiotic states between the oral microbial populations and the host immune response [10]. Besides, periodontal diseases receive several classifications, out of which two forms are commonly found in the clinic, the chronic and the aggressive periodontitis forms ( $\mathrm{CP}$ and $\mathrm{AgP}$, respectively). The main feature of $\mathrm{CP}$ is the slow and swift progression of disease that reaches subjects with an increased mean 
of age [11] and AgP has the faster linear pattern of tissue destruction with early age of onset [12]. The disease has increased prevalence in the United States affecting around $42.2 \%$ of the population [13] and young populations ranging from $0.66 \%$ in Argentina to $5.9 \%$ in Israel [14].

On the other hand, SLE is an autoimmune immune disease with multifactorial aetiology. The exact pathogenesis of SLE is still unclear. However, the apoptosis/autophagy plus clearance defects are appointed as events that result in increased exposure of nuclear self-antigens, with the activation of type 1 interferon pathway and altered immune cells signalling and autoimmune response [3]. The prevalence of the disease increased in Korea in 2015 (from $21.25 / 100,000$ people to $35.45 / 100,000$ people), by the contrary the incidence has been decreased in this population [15] what these findings are according to data of the prevalence rate from United States between 2009 and 2016 [16].

These diseases are complex and multifactorial, which one of the influencer genetic factor in their pathogeneses is the rs333 polymorphism in the CC chemokine receptor type five (CCR5). CCR5 is responsible for the events of migration immune cells and the mediation of inflammatory responses [17]. The CCR5 gene is located at the short arm position 21 on chromosome 3, denoted as 3p21 and has been described by Report et al. [18].

Diverse types of cells express this G-protein coupled chemokine receptor such as dendritic cells, macrophages as well as memory T-cells. Besides, epithelial cells, endothelial cells; fibroblasts, vascular smooth muscle and microglia, astrocytes and neurons also express CCR5 in their surface [19]. In addition, CCR5 is a co-receptor recognized by the Human Immunodeficiency Virus-1 (HIV-1) for entry into the cells.

The rs333 polymorphism in the CCR5 gene, also known as CCR5 $\triangle 32$ 32-base-pair deletion, is able to create a disruption in the CCR5 protein consequently be important to prevent the HIV-1 infection [20]. This genetic variant also was considered as protective factor against the Hepatitis B Virus (HBV)/HIV co-infection [21] and has no influence on the risk of Hepatitis $C$ Virus (HCV) and HCV/HIV co-infection [22].

The rs333 polymorphism has been related to the pathogenesis of several conditions beyond viral diseases such as celiac disease [23] and breast cancer [24]. Regarding the results on the rs333 polymorphism in the CCR5 gene and IBD, periodontitis and SLE, the data showed contradictories, which a better evaluation is required.

Therefore, when we considered the heterogeneous prevalence profile of the rs333 polymorphism among the populations [25] and the contrary results on the relation among this genetic variant and the risk of IBD, periodontitis and SLE, we attempted to perform a metaanalysis to gather these contrary results and bring a new evaluation on these data.

\section{MATERIAL AND METHODS}

In order to delineate and performing of this study, the authors have followed the Preferred Reporting Items for Systematic Reviews and Meta-Analyses (PRISMA) guidelines [26].

This meta-analysis is registered in PROSPERO database with the following assigned number: CRD42020170583.

\section{Eligibility Criteria}

The following inclusion criteria were respected to the collection process in the literature search: (1) casecontrol studies or replication genetic studies; (2) the case-patients have been diagnosed by a rigorous clinical evaluation for [I] Inflammatory Bowel Disease [27], Periodontitis (and its diverse clinical manifestations) [28] or Systemic Lupus Erythematosus [29]; (3) the control patients have presented healthy periodontal evaluation or non-systemic sign of autoimmune disease; (4) the genotypic frequency have been completely documented; (5) the studies have strictly included human beings; (6) the included participants (case and control patients) have not presented cardiovascular diseases or pregnancy.

\section{Strategy Search}

The strategy search in the literature has been performed by two authors independently in the Google Scholar, MedLine, Pubmed, WAFANG and Web of Science. The literature retrieves in these medical and scientific databases occurred for studies published before June 22, 2020. The following combination of keywords was used in the search: "periodontitis or periodontal disease or chronic periodontitis or aggressive periodontitis or inflammatory bowel disease or ulcerative colitis or Crohn's disease or systemic 
lupus erythematosus" and "polymorphism or genetic variation" and "CCR5" and "rs333 or $\Delta 32$ deletion". There was no language restriction in the strategy search. In addition, there was a screening on the references from the included articles to detect any potential additional study.

\section{Data Collection Process}

Three investigators collected the data following a standardized formulary that composed the table of characteristics of included studies presented in the results. The data were extracted by author, year of publication, ethnicity/country of the included participants, study design, clinical diagnosis of the diseases, subject type evaluated in the included studies, sample size, mean of age of the participants, respect to the Hardy-Weinberg Equilibrium (HWE), method of genotyping and the data for risk of bias assessment that will be described at soon.

\section{Risk of Bias Assessment}

To verify the methodological quality the New-Castle Ottawa Scale (NOS) was used for evaluation of included genetic studies on the aforementioned polymorphism and the relation with these diseases, which the study that received less than 7 points has been excluded.

\section{Statistical Analysis}

For the calculations applied in the meta-analysis two statistical software were used: the Review Manager Software version 5.3 (RevMan, Nordic Cochrane Centre, The Cochrane Collaboration, 2012) for Odds Ratio (OR) and heterogeneity $\left(\mathrm{I}^{2}\right)$ calculations. The publication bias has been evaluated by the use of the Comprehensive Meta-analysis statistical software version 3.3.070 (2014).

The detection of true $I^{2}$ was measured by the Cochran's $X^{2}$ test or the chi-squared Q-based statistical test. The $I^{2}$ also was analyzed by visualization of the Funnel plot graphic to verify the heterogeneity. When the observed value of $\mathrm{I}^{2}$ showed non-significant and was defined as mild or moderate $\left(I^{2}<50 \%, P>0.05\right)$ the authors have performed the pooled OR calculation by the Fixed-effect statistical model [30]. When $I^{2}$ presented a statistically significant value and was defined as elevated $\left(I^{2}>50 \%, P<0.05\right)$ the Randomeffects statistical model [31] was used for obtaining the OR calculations. The $P$ value $<0.05$ was considered as significant in all calculations. Based on mutant allele as
$\Delta 32$ and the wild type allele as $\mathrm{w}$, six genetic models were calculated. This calculation aimed to highlight the exact influence of the rs333 polymorphism in the CCR5 gene and the risk of the diseases. Then, the allele model ( $\Delta 32$ versus $\mathrm{w}$ and $\mathrm{w}$ versus $\Delta 32$ ), the dominant homozygous model $(\Delta 32 \Delta 32$ versus $w w)$, the recessive homozygous model (ww versus $\Delta 32 \Delta 32$ ) and the codominant models $(\Delta 32 \Delta 32$ versus $w w+w \Delta 32$ and $w \Delta 32$ versus $w w+\Delta 32 \Delta 32$ ) were evaluated. In addition, a sensitive analysis was performed omitting one study at a time in the pooled OR calculation for the mutant allele to detect any single interference. The authors have performed a sensitive analysis by omitting one single included study at the time to verify any possible significant change in the OR value. To detect the publication bias, the Begg's test [32] and Egger's linear regression test [33] were used as a statistical test to identify the potential publication bias (with $\mathrm{P}<0.05$ ). In addition, asymmetry in the Funnel plot for publication bias was also considered to validate the results on Begg's test and Egger's test. The data from the included studies were dichotomous and have been expressed as OR with $95 \%$ of confidence intervals $(\mathrm{Cl})$ to verify the association among the rs333 polymorphism and IBD, periodontitis and SLE.

\section{RESULTS}

\section{Characteristics of the Included Studies}

At the final of the systematic search, 19 articles with 21 case/control studies have been included after the steps of identification, screening and eligibility as showed in Figure 1. The studies were performed in IBD ( $\mathrm{n}=9$ studies), periodontitis ( $\mathrm{n}=5$ studies) and SLE ( $\mathrm{n}$ = 7 studies) and have been published between 2001 and 2017 in diverse ethnical groups from different countries. In the studies, IBD was classified as UC CD sub-types. On the other hand, periodontitis was classified as Chronic Periodontitis (CP), Aggressive Periodontitis (AgP) or Generalized Aggressive Periodontitis (GAgP). All included studies have respected the Hardy-Weinberg Equilibrium. Additional and relevant details are available in Table 1.

\section{Meta-Analysis}

\section{rs333 Polymorphism and Inflammatory Diseases}

The data available in the studies show a decreased $\Delta 32$ allele frequency, which several studies did not find double-positive participants for this allele in case or control groups (Table 1). Overall, based on 19 papers composed by 21 studies [34-52] in 4,304 case patients 


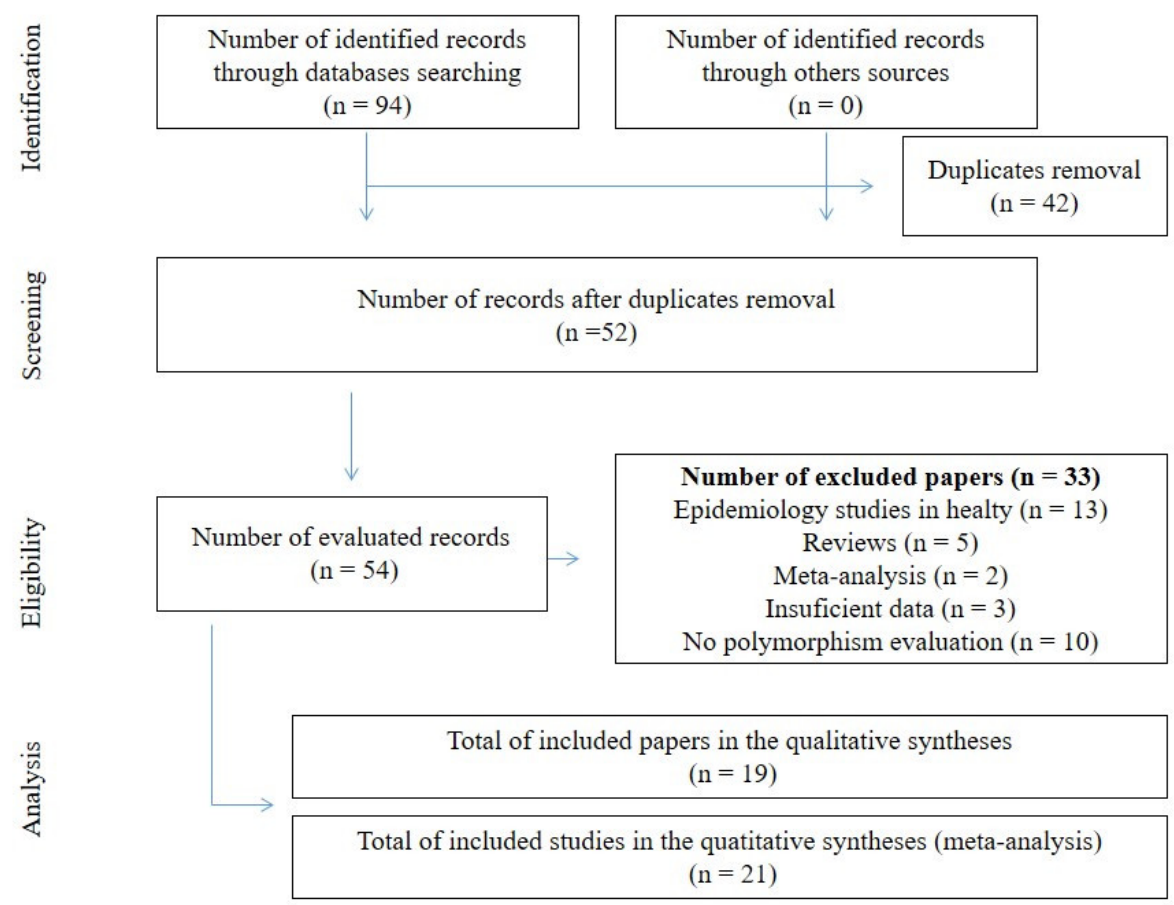

Figure 1:

and 3,492 controls, the rs333 polymorphism was not associated to any inflammatory condition approached in this systematic review and meta-analysis (Table 2 and Figure 2).

\section{Rs333 Polymorphism and Inflammatory Bowel Disease}

Nine case/control studies with 2,622 patients with a diagnosis of IBD and 2,085 controls described the relationship between the rs333 polymorphism and IBD risk [34-42]. As showed in Figure 2 the statistical evaluation showed that the $\Delta 32$ allele was not significantly associated with IBD risk when compared with the wild type allele $(\mathrm{OR}=1.05,95 \% \mathrm{Cl}: 0.91-1.20$, $\mathrm{P}=0.51)$ with non-interference of obvious heterogeneity $\left(I^{2}=0 \%\right.$, Pheterogeneity $\left.=0.71\right)$ (Figure 2A). Non-significant associations have been observed in any calculations obtained in this current metaanalysis.

\section{rs333 Polymorphism and Periodontitis}

Four papers composed of five studies aimed to determine the association between the rs333 polymorphism in CCR5 gene and periodontitis [43-46]. Interesting to note is that the meta-analysis showed the $\Delta 32$ allele as a protecting factor for periodontitis risk which the variant allele was associated with the control group but with no statistical significance $(O R=0.86$, 95\% Cl: 0.64-1.17, $\mathrm{P}=0.34$ ) (Figure 2B). On the other hand, the wild type allele was not significantly associated with case-patients $(\mathrm{OR}=1.16,95 \% \mathrm{Cl}$ : $0.86-1.56, P=0.34)$. The additional calculated genetic models are showed in Table $\mathbf{3}$, in which there were non-significant associations; all these calculations were obtained by the fixed-effect model.

\section{rs333 Polymorphism and SLE}

To verify the association between the rs333 polymorphism and SLE risk, six papers with seven studies are included in the quantitative analyses [4752]. The polymorphism was not significantly associated neither for the case-patients nor for the control group $(\Delta 32$ versus $\mathrm{w}-\mathrm{OR}=1.00,95 \% \mathrm{Cl}: 0.56-1.80, \mathrm{P}=$ 1.00 , for both calculations) (Figure $2 \mathrm{C}$ ). The same results for the variant allele and the wild type are curious and should be noted, especially for the increased heterogeneity value $\left(I^{2}=76 \%, P<0.0003\right)$ what led us to use the random-effects statistical model for these calculations. Other important calculations are showed in Table 4.

\section{Sensitive Analysis and Publication Bias}

The sensitive analysis has indicated that no single study changed the pooled OR value what led us to take these data as accurate. Likewise, the results on the Begg's test and Egger's linear regression test did not find any evidence of possible publication bias (Table 5), which is attested by the absence of obvious asymmetry in the funnel plots (Figure 3), with consequent validation of our results. 


\begin{tabular}{|c|c|c|c|c|c|c|c|c|c|c|c|c|c|c|c|c|c|c|c|}
\hline \multicolumn{2}{|l|}{$\frac{n}{2}$} & $\Lambda$ & N & $r$ & $\infty$ & $\wedge$ & $\infty$ & $\infty$ & $\infty$ & r & $\infty$ & $\infty$ & r & $r$ & $r$ & $\Lambda$ & $\wedge \sim$ & N & N \\
\hline \multicolumn{2}{|c|}{ 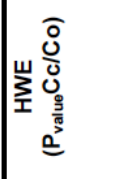 } & $\bar{z}$ & 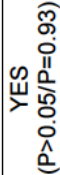 & 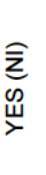 & 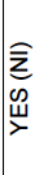 & $\begin{array}{l}\overline{\bar{z}} \\
\stackrel{\mathbb{W}}{\nu}\end{array}$ & $\overline{\mathbf{z}}$ & 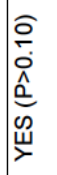 & 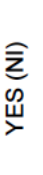 & $\bar{z}$ & 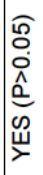 & $\bar{z}$ & 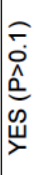 & $\overline{\mathbf{z}}$ & 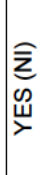 & 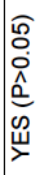 & 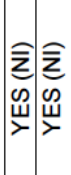 & 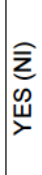 & $\begin{array}{l}\overline{\bar{z}} \\
\stackrel{\mu}{\nu}\end{array}$ \\
\hline \multirow{3}{*}{ 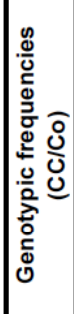 } & 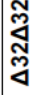 & 新 & $\tilde{N}$ & 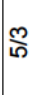 & $\stackrel{2}{\aleph}$ & $\stackrel{?}{?}$ & $\delta$ & $\underset{⿱}{\stackrel{ \pm}{*}}$ & 芯 & $\stackrel{m}{\circ}$ & 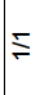 & $\overline{\text { N }}$ & ని & $\stackrel{9}{9}$ & $\stackrel{?}{9}$ & กี & $\mathbb{N} \mid \frac{\mathfrak{N}}{\sim}$ & $\stackrel{?}{?}$ & 응 \\
\hline & $\tilde{\mathscr{U}}_{3}$ & 忍 & 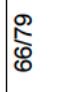 & 忍 & 莺 & $\stackrel{\infty}{\Sigma}$ & 蛋 & 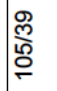 & ֻু & 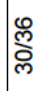 & $\frac{\bar{m}}{\bar{m}}$ & $\underset{n}{\text { N }}$ & 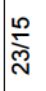 & 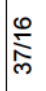 & 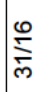 & 苾 & 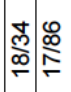 & 商 & $\bar{\sigma}$ \\
\hline & $\xi$ & $\begin{array}{l}\stackrel{\circ}{\infty} \\
\text { 商 } \\
\text { N }\end{array}$ & 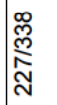 & 离 & 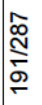 & $\frac{2}{2}$ & 㗹 & 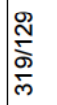 & 誉 & 雚 & $\begin{array}{l}\mathscr{\infty} \\
\stackrel{\infty}{0} \\
\stackrel{\leftrightarrow}{N}\end{array}$ & $\begin{array}{l}\mathscr{Q} \\
\frac{2}{2} \\
\hat{0}\end{array}$ & 志 & $\frac{d}{\frac{8}{2}}$ & 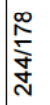 & 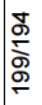 & 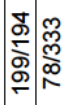 & 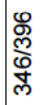 & 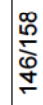 \\
\hline
\end{tabular}

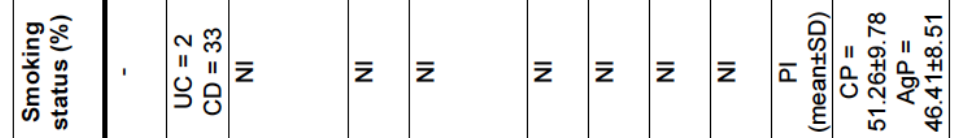

\begin{tabular}{|l|l|l|l|l|l|l|l|l|l|l|}
\hline 0 & & & & & & & & & & \\
\hline
\end{tabular}

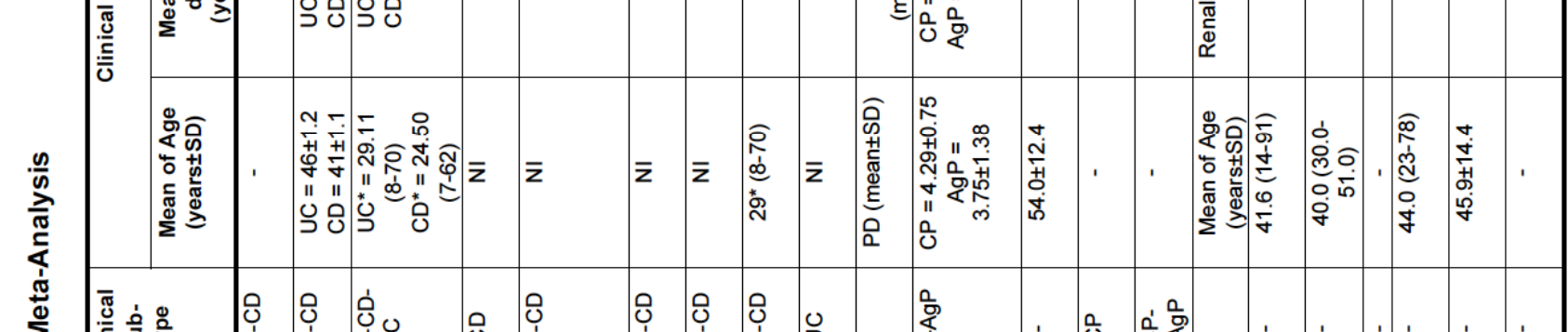

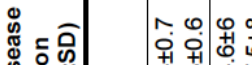

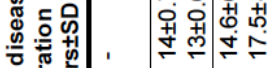

管

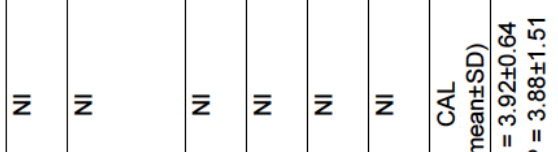

$\mid \begin{array}{lll}11 \\ 0 & 0 \\ 0 & 0\end{array}$ 응

कू

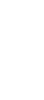
产

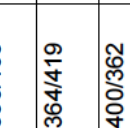
旁 结路 ป

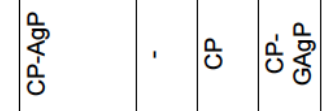

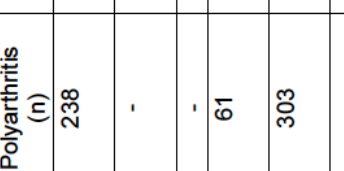

\begin{tabular}{|c|c|c|c|c|c|c|c|c|c|c|c|c|c|c|c|c|c|c|}
\hline 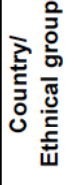 & 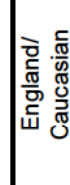 & 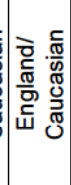 & 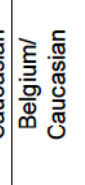 & 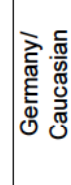 & 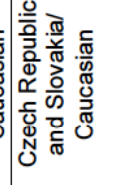 & 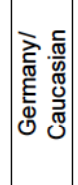 & 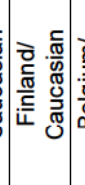 & 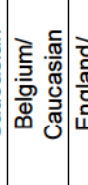 & 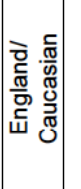 & 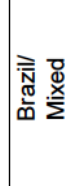 & 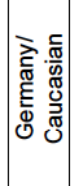 & 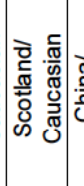 & 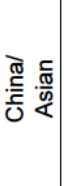 & 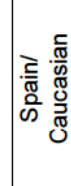 & 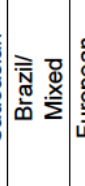 & 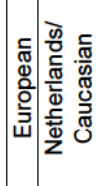 & 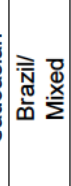 & 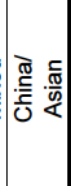 \\
\hline 离 & స్తి & ষั & ֻั & ర్సે & ֻ ڤై & ষ্ণ & $\bar{్}$ & $\bar{\sim}$ & : & సิ & ్ֻని & స్రి & ডั̀ & ఇి & $\stackrel{n}{\stackrel{n}{2}}$ & 龍을 & 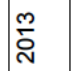 & ֻ ֻ \\
\hline 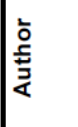 & | & 况 & 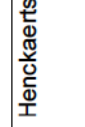 & 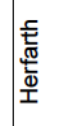 & 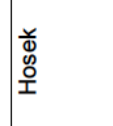 & $\begin{array}{l}\frac{c}{\bar{t}} \\
\text { 而 }\end{array}$ & \begin{tabular}{|l|l}
$\frac{\pi}{0}$ \\
0 \\
0 \\
0 \\
0 \\
0 \\
0
\end{tabular} & 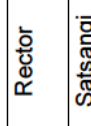 & | & 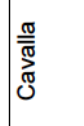 & 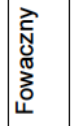 & 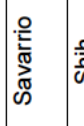 & 常 & 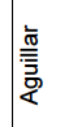 & 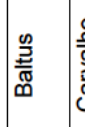 & 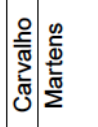 & 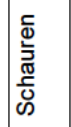 & 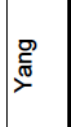 \\
\hline 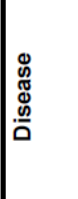 & 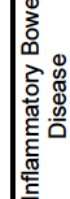 & & & & & & & & & 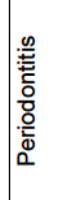 & & & & 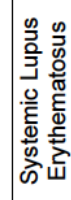 & & & & \\
\hline
\end{tabular}

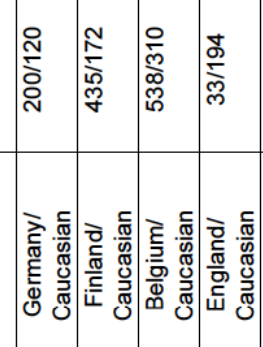

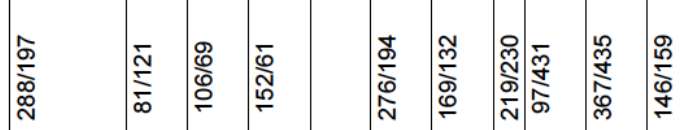


Table 2: Meta-Analysis of Comparison to rs333 Polymorphism in CCR5 Gene and Inflammatory Bowel Disease Risk (Allelic and Genotypic Comparisons)

\begin{tabular}{|c|c|c|c|c|c|c|}
\hline & $\begin{array}{c}\text { Comparison } \\
\text { (n) }\end{array}$ & OR $(95 \% \mathrm{CI})$ & $\begin{array}{c}\text { Z value test } \\
\text { (P value) }\end{array}$ & $I^{2}(\%)$ & Pheterogeneity & $\begin{array}{l}\text { Statistical } \\
\text { model used }\end{array}$ \\
\hline & $\operatorname{IBD}(n=9)$ & & & & & \\
\hline $\begin{array}{l}\text { Allelic } \\
\text { models }\end{array}$ & w versus $\Delta 32$ & $0.96(0.84-1.09)$ & $0.66(0.51)$ & 0 & 0.71 & $\mathrm{~F}$ \\
\hline $\begin{array}{c}\text { Homozygous } \\
\text { dominant } \\
\text { model }\end{array}$ & $\Delta 32 \Delta 32$ versus $w w$ & $0.81(0.47-1.42)$ & $0.73(0.47)$ & 0 & 0.91 & $\mathrm{~F}$ \\
\hline $\begin{array}{c}\text { Recessive } \\
\text { homozygous } \\
\text { model }\end{array}$ & ww versus $\Delta 32 \Delta 32$ & $0.93(0.81-1.08)$ & $0.93(0.35)$ & 7 & 0.38 & $\mathrm{~F}$ \\
\hline \multirow{2}{*}{$\begin{array}{l}\text { Codominant } \\
\text { models }\end{array}$} & $\Delta 32 \Delta 32$ versus $w w+\Delta 32 w$ & $0.80(0.46-1.39)$ & $0.79(0.43)$ & 0 & 0.87 & $\mathrm{~F}$ \\
\hline & $\Delta 32 w$ versus $w w+\Delta 32 \Delta 32$ & $1.09(0.94-1.27)$ & $1.16(0.25)$ & 31 & 0.17 & $\mathrm{~F}$ \\
\hline
\end{tabular}

IBD - Inflammatory Bowel Disease, OR - Odds Ratio, $\mathrm{Cl}$ - Confidence Intervals, $\mathrm{I}^{2}$ - Heterogeneity, $\Delta 32-$ mutant allele, m - wild type allele, $\mathrm{F}$ - Fixed-effect statistical model.

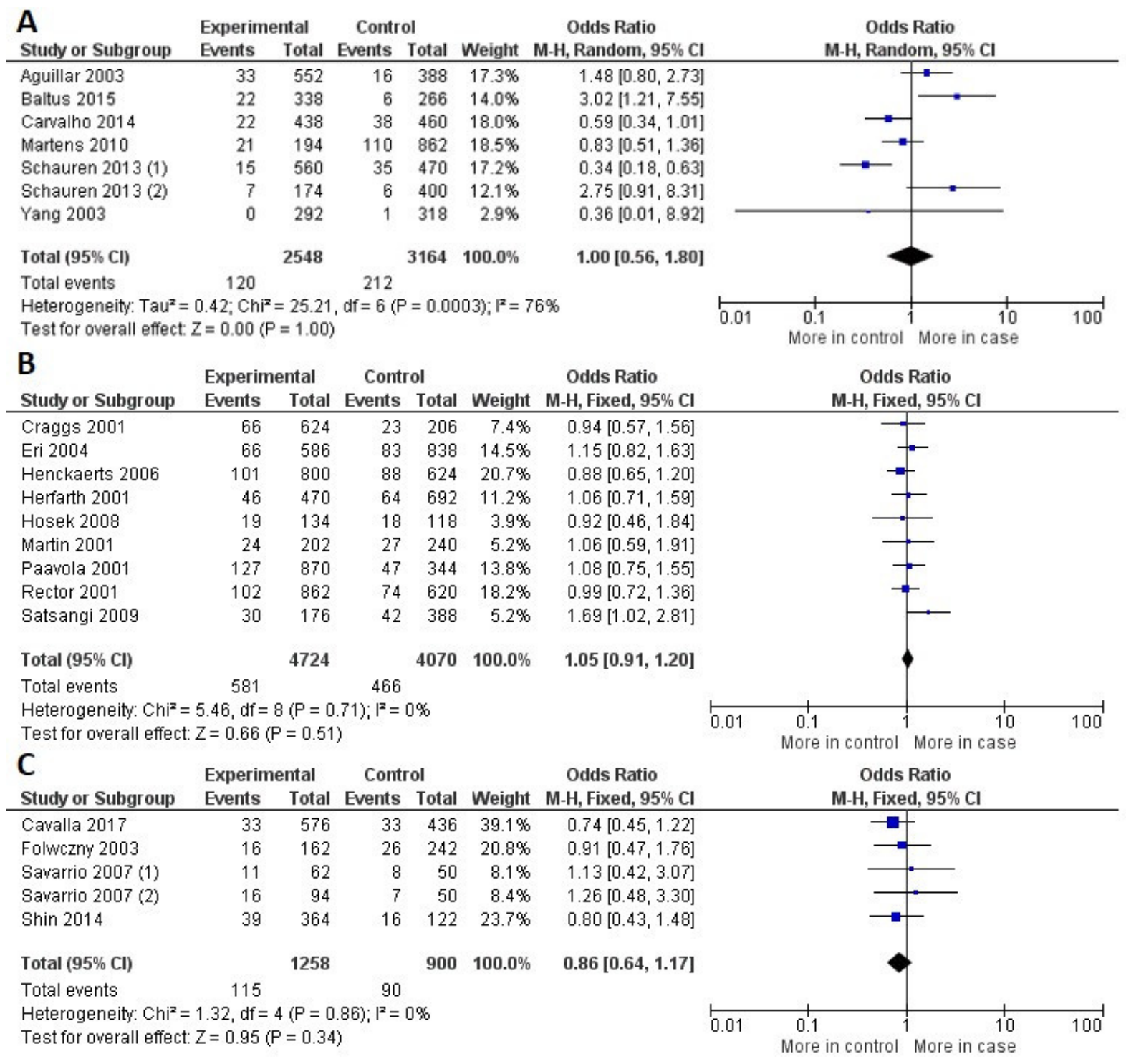

Figure 2: 
Table 3: Meta-Analysis of Comparison to rs333 Polymorphism in CCR5 Gene and Periodontitis risk (Allelic and Genotypic Comparisons)

\begin{tabular}{|c|c|c|c|c|c|c|}
\hline & $\begin{array}{c}\text { Comparison } \\
\text { (n) }\end{array}$ & OR $(95 \% \mathrm{CI})$ & $\begin{array}{c}\text { Z value test } \\
\text { (P value) }\end{array}$ & $I^{2}(\%)$ & Pheterogeneity & $\begin{array}{l}\text { Statistical } \\
\text { model used }\end{array}$ \\
\hline & Periodontitis $(n=5)$ & & & & & \\
\hline $\begin{array}{l}\text { Allelic } \\
\text { models }\end{array}$ & w versus $\Delta 32$ & $1.16(0.86-1.56)$ & $0.95(0.34)$ & 0 & 0.86 & $\mathrm{~F}$ \\
\hline $\begin{array}{c}\text { Homozygous } \\
\text { dominant } \\
\text { model }\end{array}$ & $\Delta 32 \Delta 32$ versus $w w$ & $1.67(0.42-6.64)$ & $0.73(0.47)$ & 0 & 0.86 & $\mathrm{~F}$ \\
\hline $\begin{array}{c}\text { Recessive } \\
\text { homozygous } \\
\text { model }\end{array}$ & ww versus $\Delta 32 \Delta 32$ & $0.60(0.15-2.39)$ & $0.73(0.47)$ & 0 & 0.86 & $\mathrm{~F}$ \\
\hline \multirow{2}{*}{$\begin{array}{l}\text { Codominant } \\
\text { models }\end{array}$} & $\Delta 32 \Delta 32$ versus $w w+\Delta 32 w$ & $1.74(0.44-6.93)$ & $0.79(0.43)$ & 0 & 0.86 & $\mathrm{~F}$ \\
\hline & $\Delta 32 w$ versus $w w+\Delta 32 \Delta 32$ & $0.77(0.55-1.07)$ & $1.59(0.11)$ & 0 & 0.95 & $\mathrm{~F}$ \\
\hline
\end{tabular}

OR - Odds Ratio, $\mathrm{Cl}$ - Confidence Intervals, $\mathrm{I}^{2}$ - Heterogeneity, $\Delta 32$ - mutant allele, $\mathrm{m}$ - wild type allele, $\mathrm{F}$ - Fixed-effect statistical model.

Table 4: Meta-Analysis of Comparison to rs333 Polymorphism in CCR5 Gene Systemic Lupus Erythematous Risk (Allelic and Genotypic Comparisons)

\begin{tabular}{|c|c|c|c|c|c|c|}
\hline & $\begin{array}{c}\text { Comparison } \\
\text { (n) }\end{array}$ & OR $(95 \% \mathrm{Cl})$ & $\begin{array}{c}\text { Z value test } \\
\text { (P value) }\end{array}$ & $\mathrm{I}^{2}(\%)$ & Pheterogeneity & $\begin{array}{l}\text { Statistical } \\
\text { model used }\end{array}$ \\
\hline & $\operatorname{SLE}(n=7)$ & & & & & \\
\hline $\begin{array}{l}\text { Allelic } \\
\text { models }\end{array}$ & w versus $\Delta 32$ & $1.00(0.56-1.80)$ & $0.00(1.00)$ & $76 \%$ & 0.0003 & $\mathrm{R}$ \\
\hline $\begin{array}{c}\text { Homozygous } \\
\text { dominant } \\
\text { model }\end{array}$ & $\Delta 32 \Delta 32$ versus ww & $0.96(0.41-2.28)$ & $0.08(0.93)$ & 0 & 0.60 & $\mathrm{~F}$ \\
\hline $\begin{array}{c}\text { Recessive } \\
\text { homozygous } \\
\text { model }\end{array}$ & ww versus $\Delta 32 \Delta 32$ & $1.04(0.44-2.45)$ & $0.08(0.93)$ & 0 & 0.60 & $\mathrm{~F}$ \\
\hline \multirow{2}{*}{$\begin{array}{l}\text { Codominant } \\
\text { models }\end{array}$} & $\Delta 32 \Delta 32$ versus $w w+\Delta 32 w$ & $0.99(0.42-2.34)$ & $0.02(0.98)$ & 0 & 0.64 & $\mathrm{~F}$ \\
\hline & $\Delta 32 \mathrm{w}$ versus $w w+\Delta 32 \Delta 32$ & $0.93(0.53-1.63)$ & $0.25(0.81)$ & 70 & 0.003 & $\mathrm{R}$ \\
\hline
\end{tabular}

SLE - Systemic Lupus Erythematous, OR - Odds Ratio, $\mathrm{Cl}$ - Confidence Intervals, $\mathrm{I}^{2}$ - Heterogeneity, $\Delta 32$ - mutant allele, $\mathrm{m}$ - wild type allele, $\mathrm{R}$ - Random-effects statistical model, F - Fixed-effect statistical model.

Table 5: P-Value for the Begg's Test and Egger's Linear Regression Test for the Allelic Dominant Model Evaluation in this Current Meta-Analysis

\begin{tabular}{|c|c|c|c|}
\hline Disease & Comparison & Begg's test & Egger's linear regression test \\
\hline \hline IBD & \multirow{2}{*}{ s32 versus w } & 0.754 & 0.479 \\
\cline { 1 - 3 } Periodontitis & & 0.082 & 0.074 \\
\cline { 3 - 4 } SLE & & 0.763 & 0.503 \\
\hline
\end{tabular}

IBD - Inflammatory Bowel Disease, SLE - Systemic Lupus Erythematous, $\Delta 32$ - Mutant allele, w - Wild-type allele.

\section{DISCUSSION}

This current meta-analysis is the first to attempt to verify the relationship between the rs333 polymorphism in CCR5 gene and the risk of these inflammatory conditions. Despite the statistical results showed nonsignificant associations in any allelic or genotypic evaluations our results are accurate by the decreased heterogeneity value in most calculations, the absence of publication bias and the evaluation of adjustment factors for the evaluated inflammatory illness.

The CCR5 is a relevant receptor described in the literature, as the driver of cells for inflammatory sites 
A

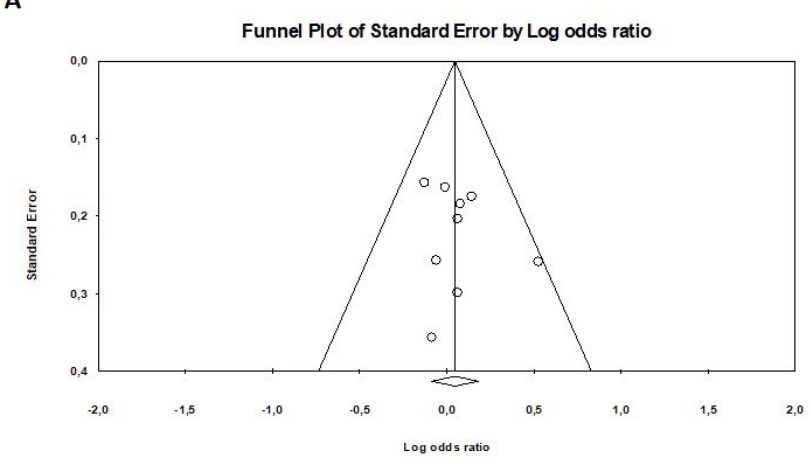

B

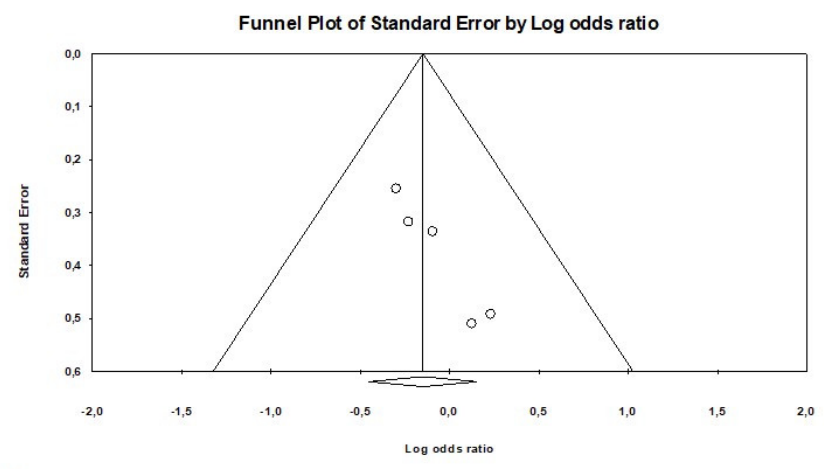

C

Funnel Plot of Standard Error by Log odds ratio

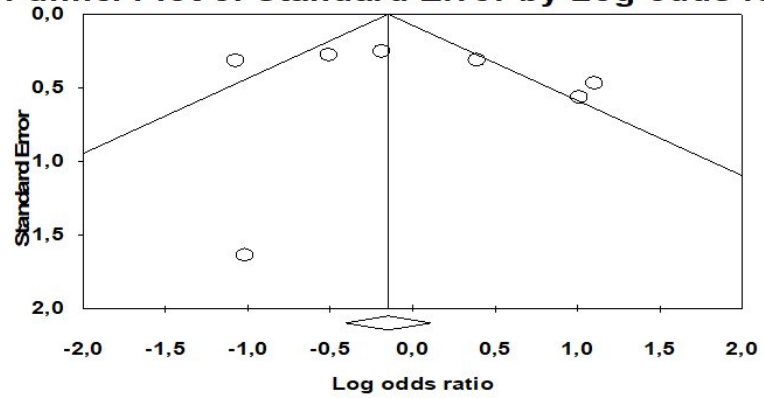

Figure 3:

[53] and, in assembly with other chemokine receptors, be able to enhance the T-cells functions [54]. Besides, CCR5 plays an important role in macrophage action, either by the increased responsiveness of monocytederived macrophages [55] or by the induction of macrophage survival during viral infection by antiapoptotic signals [56]. It is well established that macrophages are critical cells for the initiation of the inflammatory process in IBD [57], periodontitis [58] and SLE [59].

In genetic levels, the rs333 polymorphism is another factor that influences the role of CCR5 in inflammatory conditions. Previous authors suggested that this gene variation is generated by the occurrence of stem-loop structure in DNA [60] which the $\Delta 32$ deletion leads to a synthesis of a truncated protein that fails to be inserted into the cell membrane, becomes nonfunctional [61]. This genetic variation is exhaustively associated with HIV-1 resistance by the decreased or absent expression of this co-receptor for virus-cell infection [62-64] and also with others virus type infections that were approached in previous studies $[65,66]$.

On inflammatory conditions risk, the rs333 polymorphism was related to the risk of rheumatoid arthritis [67], multiple sclerosis [68] and psoriasis [69]. The data on IBD risk, studies bring contrary results, which some authors did not find the correlation between the delta32 allele with UC or CD [34] and other authors identified a significant association of this polymorphism with control groups with a possible protective effect [36]. The results from our metaanalysis are in disagreement with this previous finding and showed the non-significant association between the variant allele with the IBD risk $(P>0.05)$. One explanation for this finding is the pooled data from the meta-analytic calculations that increase the number of case/control elements and increases the power of association.

The non-significant between the polymorphism and IBD risk also was found for periodontitis and SLE (Table 2). It is relevant to denote two contradictory points in these results. First, the number of included studies for the diseases ( $n=5$ and $n=7$, respectively) might promote a bias in the interpretation of results due to limited statistical power of noteworthiness association or biased value of $\mathrm{I}^{2}[70]$.

On the other hand, periodontitis and SLE showed as two-way relationship diseases [71]. This two-way relationship may be explained by the constant interaction between the oral human microbiome and the host, which an imbalance leads to bone resorption with a periodontal dysbiosis that goes beyond the oral compartment influencing the SLE pathogenesis [72]. Although there was a non-significant association between the rs1333 polymorphism in the CCR5 gene for these two conditions, the results may be lead with caution.

Although to the best of our knowledge, this is the first meta-analysis that aimed to determine the association among the rs 1333 polymorphism in CCR5 gene and IBD, periodontitis and SLE, which results in bringing the relevant data and the meta-analysis showed important limitations that should be appointed.

First, the decreased number of selected studies did not show robustness in the results what may be 
considered as a source of bias. To avoid the potential bias of this limitation we have used accurate and robust statistical methods to validate our results. Second, important features from the evaluated patients were not available in the included articles. A complete evaluation of adjusting factors such as familiar history, gender, smokers and non-smokers, stratified age data and others conditions that influence the development of the diseases was not possible due to the limited information in the papers. Third, these three inflammatory diseases are clinical conditions that receive several classifications. An evaluation based on these clinical variations could not be performed due to limited data available in the studies. Fourth, a large number of the meta-analysis calculations were influenced by a significant $\mathrm{I}^{2}$ and by the use of Random-effects as a statistical model. The value of $\mathrm{I}^{2}$ indicates how the studies are inconsistent in a statistical manner. It may be a relevant factor in the meta-analysis because the true heterogeneity may affect directly the statistical model used on the results. The Random-effects statistical model provides an increased weight to studies containing a smaller sample size, which is not considered as totally trustworthy. Fifth, diverse non-significant associations were found in the meta-analysis calculations. However, the non-significant $\mathrm{P}$-value does not always reflect the absence of clinical relevance. Sixth, we have evaluated one single polymorphism, other genetic variations may influence the rs1333 role in these inflammatory conditions. Previous studies available in the literature already showed the considerable impact of genetic polymorphisms and IBD [73], periodontitis [74-79] and SLE $[80,81]$ further studies are required to validate our results.

\section{CONCLUSION}

In conclusion, this current meta-analysis composed of 21 case-control studies showed the non-significant association among the rs1333 polymorphism in CCR5 gene and IBD, periodontitis and SLE.

\section{FUNDING}

None.

\section{LIST OF ABBREVIATIONS}

rs333 = Reference Sequence polymorphism in the CCR5 gene

CCR5 = $\mathrm{CC}$ chemokine receptor type five
IBD
$=$ Inflammatory Bowel Disease
SLE = Systemic Lupus Erythematous
$\mathrm{I}^{2} \quad=$ Heterogeneity
OR $=$ Odds Ratio
$\mathrm{Cl}=$ Confidence Intervals
$\mathrm{CP}=$ Chronic Periodontitis
AgP $=$ Aggressive Periodontitis
CD $=$ Cronh's Disease
UC $\quad=$ Ulcerative Colitis
HIV-1 = Human Immunodeficiency Virus-1
HBC = Hepatitis $B$ Virus
$\mathrm{HCV}=$ Hepatitis $\mathrm{C}$ Virus
PRISMA = Preferred Reporting Items for Systematic Reviews and Meta-Analyses
HWE = Hardy-Weinberg Equilibrium
NOS $=$ New-Castle Ottawa Scale
$\mathrm{GAgP}=$ Generalized Aggressive Periodontitis

\section{ACKNOWLEDGEMENTS}

None.

\section{REFERENCES}

[1] Loos BG, Van Dyke TE. The role of inflammation and genetics in periodontal disease. Periodontol 2000 2020; 83(1): 26-39.

https://doi.org/10.1111/prd.12297

[2] Bianco AM, Girardelli M, Tommasini A. Genetics of inflammatory bowel disease from multifactorial to monogenic forms. World J Gastroenterol 2015; 21: 12296-310. https://doi.org/10.3748/wjg.v21.i43.12296

[3] Deng Y, Tsao BP. Updates in lupus genetics. Curr Rheumatol Rep 2017; 19: 68 https://doi.org/10.1007/s11926-017-0695-Z

[4] Zuo T, Kamm MA, Colombel JF, et al. Urbanization and the gut microbiota in health and inflammatory bowel disease. Nat Rev Gastroenterol Hepatol 2018; 15: 440-52. https://doi.org/10.1038/s41575-018-0003-z

[5] Eppinga $\mathrm{H}$, Poortinga $\mathrm{S}$, Thio $\mathrm{HB}$, et al. Prevalence and phenotype of concurrent psoriasis and inflammatory bowe disease. Inflamm Bowel Dis 2017; 23: 1783-89. https://doi.org/10.1097/MIB.0000000000001169

[6] Jones GR, Lyons M, Plevris $\mathrm{N}$, et al. IBD prevalence in Lothian, Scotland, derived by capture-recapture methodology. Gut 2019; 68: 1953-60. https://doi.org/10.1136/gutjnl-2019-318936 
[7] Chang S, Shen B. Classification and Reclassification of Inflammatory Bowel Diseases: From Clinical Perspective. In: Interventional Inflammatory Bowel Disease: Endoscopic Management and Treatment of Complications. Academic Press 2018; 17-34. https://doi.org/10.1016/B978-0-12-811388-2.00002-6

[8] Younis N, Zarif R, Mahfouz R. Inflammatory bowel disease: between genetics and microbiota. Mol Biol Rep 2020; 1-11. https://doi.org/10.1007/s11033-020-05318-5

[9] Kinane DF, Stathoupoulou PG, Papapanou PN. Periodontal diseases. Nat Rev Dis Primers 2017; 3: 17038. https://doi.org/10.1038/nrdp.2017.38

[10] Silva N, Abusleme L, Bravo D, et al. Host response mechanisms in periodontal diseases. J Appl Oral Sci 2015; 23: 329-55. https://doi.org/10.1590/1678-775720140259

[11] López R, Baelum V. Periodontal disease classifications revisited. Eur J Oral Sci 2015; 123: 385-9. https://doi.org/10.1111/eos.12227

[12] Albandar JM. Aggressive periodontitis: case definition and diagnostic criteria. Periodontol 2000 2014; 65: 13-26. https://doi.org/10.1111/prd.12014

[13] Eke PI, Borgnakke WS, Genco RJ. Recent epidemiologic trends in periodontitis in the USA. Periodontol 2000; 82: 25767.

https://doi.org/10.1111/prd.12323

[14] Catunda RQ, Levin L, Kornerup I, et al. Prevalence of Periodontitis in Young Populations: A Systematic Review. Oral Health Preventive Dent 2019; 17: 195-202.

[15] Bae, EH, Lim SY, Han KD, et al. Trend of prevalence and incidence of systemic lupus erythematosus in South Korea, 2005 to 2015: a nationwide population-based study. Korean J Intern Med 2020; 35: 652-61. https://doi.org/10.3904/kjim.2018.303

[16] Li S, Gong T, Peng Y, et al. Prevalence and incidence of systemic lupus erythematosus and associated outcomes in the 2009-2016 US Medicare population. Lupus 2020; 29: 15-26. https://doi.org/10.1177/0961203319888691

[17] Ellwanger JH, Kulmann LB, Lima KV, et al. Beyond HIV infection: neglected and varied impacts of CCR5 and CCR5 32 on viral diseases. Virus Res 2020; 286: 198040. https://doi.org/10.1016/j.virusres.2020.198040

[18] Raport CJ, Gosling J, Schweickart VL, Gray PW, Charo IF. Molecular cloning and functional characterization of a novel human CC chemokine receptor (CCR5) for RANTES, MIP-

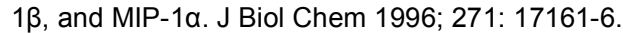
https://doi.org/10.1074/jbc.271.29.17161

[19] Rautenbach A, Williams AA. Metabolomics as an Approach to Characterise the Contrasting Roles of CCR5 in the Presence and Absence of Disease. Int J Mol Sci 2020; 21: 1472. https://doi.org/10.3390/ijms21041472

[20] Verma MK, Shakya S. Genetic variation in the chemokine receptor 5 gene and course of HIV infection; review on genetics and immunological aspect. Genes Dis 2020; 8: 47583.

https://doi.org/10.1016/j.gendis.2020.04.007

[21] Ellwanger JH, Kulmann LB, Wolf JM, et al. Role of the genetic variant CCR5 $\triangle 32$ in HBV infection and HBV/HIV coinfection. Virus res 2020; 277: 197838. https://doi.org/10.1016/j.virusres.2019.197838

[22] Ellwanger $\mathrm{JH}$, Leal BK, Valverde VJM, et al. CCR5 $\Delta 32$ in HCV infection, HCV/HIV co-infection, and HCV-related diseases. Infect Genet Evol 2018; 59: 163-6. https://doi.org/10.1016/j.meegid.2018.02.002

[23] Słomiński B, Ławrynowicz U, Myśliwska J, et al. CCR5- $\Delta 32$ gene polymorphism is related to celiac disease and autoimmune thyroiditis coincidence in patients with type 1 diabetes. J Diabetes Complications 2017; 31: 615-8. https://doi.org/10.1016/j.jdiacomp.2016.10.031
[24] Fatima F, Saleem S, Hameed A, et al. Association analysis and allelic distribution of deletion in CC chemokine receptor 5 gene (CCR5 $\Delta 32)$ among breast cancer patients of Pakistan. Mol Biol Rep 2018; 46: 2387-94.

https://doi.org/10.1007/s11033-019-04699-6

[25] Solloch UV, Lang K, Lange V, Böhme I, Schmidt AH, Sauter $\mathrm{J}$. Frequencies of gene variant CCR5- $\triangle 32$ in 87 countries based on next-generation sequencing of 1.3 million individuals sampled from 3 national DKMS donor centers. Human Immunol 2017; 78: 710-7. https://doi.org/10.1016/j.humimm.2017.10.001

[26] Moher D, Liberati A, Tetzlaff J, et al. Preferred reporting items for systematic reviews and meta-analyses: the PRISMA statement (Chinese edition). Chin J Integr Med 2009; 07: 889-96.

https://doi.org/10.3736/jcim20090918

[27] Levine A, Koletzko S, Turner D, et al. ESPGHAN revised porto criteria for the diagnosis of inflammatory bowel disease in children and adolescents. J Pediatr Gastroenterol Nutr 2014; 58: 795-806.

https://doi.org/10.1097/MPG.0000000000000239

[28] Page RC, Eke PI. Case definitions for use in population-based surveillance of periodontitis. J Periodontol 2007; 78: 1387-99. https://doi.org/10.1902/jop.2007.060264

[29] Yu C, Gershwin ME, Chang C. Diagnostic criteria for systemic lupus erythematosus: a critical review. J Autoimmun 2014; 48: 10-3.

https://doi.org/10.1016/j.jaut.2014.01.004

[30] Mantel N, Haenszel W. Statistical aspects of the analysis of data from retrospective studies of disease. J Natl Cancer Inst 1959; 22: 719-48.

[31] Dersimonian R, Laird N. Meta-analysis in clinical trials. Control Clin Trials 1986; 7: 177-88. https://doi.org/10.1016/0197-2456(86)90046-2

[32] Begg CB, Mazumdar M. Operating characteristics of a rank correlation test for publication bias. Biometrics 1994; 1088101.

https://doi.org/10.2307/2533446

[33] Egger M, Davey Smith G, Schneider M, Minder C. Bias in meta-analysis detected by a simple, graphical test. Bmj 1997; 315: 629-34. https://doi.org/10.1136/bmj.315.7109.629

[34] Craggs A, Welfare M, Donaldson PT, Mansfield JC. The CC chemokine receptor $5 \Delta 32$ mutation is not associated with inflammatory bowel disease (IBD) in NE England. Genes Immun 2001; 2: 114-6. https://doi.org/10.1038/sj.gene.6363735

[35] Eri R, Jonsson JR, Pandeya $N$, et al. CCR5- $\Delta 32$ mutation is strongly associated with primary sclerosing cholangitis. Genes Immun 2004; 5: 444-50. https://doi.org/10.1038/sj.gene.6364113

[36] Henckaerts L, Fevery J, Van SW, et al. CC-Type chemokine receptor $5-\Delta 32$ mutation protects against primary sclerosing cholangitis. Inflamm Bowel Dis 2006; 12: 272-7. https://doi.org/10.1097/01.MIB.0000209790.21737.28

[37] Herfarth H, Pollok KB, Göke M, Press A, Oppermann M. Polymorphism of CC chemokine receptors CCR2 and CCR5 in Crohn's disease. Immunol Lett 2001; 77: 113-7. https://doi.org/10.1016/S0165-2478(01)00199-7

[38] Hosek J, Bartosova L, Gregor P, et al. Frequency of representative single nucleotide polymorphisms associated with inflammatory bowel disease in the Czech Republic and Slovak Republic. Folia Biol (Praha) 2008; 54: 88-96.

[39] Martin K, Heinzlmann M, Borchers R, et al. $\Delta 32$ mutation of the chemokine-receptor 5 gene in inflammatory bowel disease. Clin Immunol 2001; 98: 18-22. https://doi.org/10.1006/clim.2000.4943 
[40] Paavola P, HelioĖ T, Kiuru M, et al. Genetic analysis in Finnish families with inflammatory bowel disease supports linkage to chromosome 3p21. Eur J Hum Genet 2001; 9: 328-34.

\section{https://doi.org/10.1038/sj.ejhg.5200626}

[41] Rector A, Vermeire S, Thoelen I, et al. Analysis of the CC chemokine receptor 5 (CCR5) delta-32 polymorphism in inflammatory bowel disease. Hum Genet 2001; 108: 190-3. https://doi.org/10.1007/s004390100462

[42] Satsangi J, Vermeire S, Thoelen I, et al. CCR5 $\Delta 32$ polymorphism in inflammatory bowel disease: further association with ulcerative colitis and with primary sclerosing cholangitis. Gastroenterol 2000; 4: A337.

https://doi.org/10.1016/S0016-5085(00)83443-5

[43] Cavalla F, Biguetti CC, Dionisio TJ, et al. CCR5 $\Delta 32$ (rs333) polymorphism is associated with decreased risk of chronic and aggressive periodontitis: a case-control analysis based in disease resistance and susceptibility phenotypes. Cytokin 2018; 103: 142-9. https://doi.org/10.1016/j.cyto.2017.09.022

[44] Folwaczny M, Glas J, Török HP, Fricke K, Folwaczny C. Prevalence of the chemokine receptor CCR5- $\triangle 32$ gene mutation in periodontal disease. Clin Immunol 2003; 109: 325-9.

https://doi.org/10.1016/j.clim.2003.08.001

[45] Savarrio L, Donati M, Carr C, Kinane DF, Berglundh T. Interleukin-24, RANTES and CCR5 gene polymorphisms are not associated with chronic adult periodontitis. J Periodontal Res 2007; 42: 152-8.

https://doi.org/10.1111/j.1600-0765.2006.00928.x

[46] Shih YS, Fu E, Fu MM, et al. Association of CCL5 and CCR5 gene polymorphisms with periodontitis in Taiwanese. J Periodontal 2014; 85: 1596-602.

https://doi.org/10.1902/jop.2014.130651

[47] Aguilar F, Núñez RA, Torres B, Wichmann I, SánchezRomán, González-Escribano MF. Chemokine receptor CCR2/CCR5 polymorphism in Spanish patients with systemic lupus erythematosus. J Rheumatology 2003; 30: 1770-774.

[48] Baltus THL, Kallaur AP, Lozovoy MAB, et al. CCR5 $\Delta 32$ (rs333) polymorphism is associated with the susceptibility to systemic lupus erythematosus in female Brazilian patients. Rheumatol int 2016; 36: 7-15. https://doi.org/10.1007/s00296-015-3308-z

[49] Carvalho C, Calvisi SL, Leal B, et al. CCR5-Delta32: implications in SLE development. Int J Immunogenet 2014; 41: 236-41.

https://doi.org/10.1111/iji.12094

[50] Martens HA, Gross S, van der Steege G, et al. Lack of association of CC chemokine receptor $5 \Delta 32$ deletion status with rheumatoid arthritis, systemic lupus erythematosus, lupus nephritis, and disease severity. J Rheumatol 2010; 37: 2226-31.

https://doi.org/10.3899/jrheum.091468

[51] Schauren JS, Marasca JA, Veit TD, et al. CCR5delta32 in systemic lupus erythematosus: implications for disease susceptibility and outcome in a Brazilian population. Lupus 2013; 22: 802-9.

https://doi.org/10.1177/0961203313491848

[52] Yang S, Ye D, Li X. The SNPs of chemokine RANTES promoter and the mutation of its' receptor CCR5 in SLE and controls in Han indigenous in Chinese. Chinese $\mathrm{J}$ Microbiol Immunol 2003; 23: 723-7.

[53] Barmania F, Pepper SM. CC chemokine receptor type five (CCR5): an emerging target for the control of HIV infection. Appl Transl Genom 2013; 2: 3-16. https://doi.org/10.1016/j.atg.2013.05.004

[54] Contento RL, Molon B, Boularan C, et al. CXCR4-CCR5: a couple modulating T cell functions. Proc Natl Acad Sci 2008; 105: 10101-6.

https://doi.org/10.1073/pnas.0804286105
[55] Kaufmann A, Salentin R, Gemsa D, Sprenger H. Increase of CCR1 and CCR5 expression and enhanced functional response to MIP-1a during differentiation of human monocytes to macrophages. J Leukoc Biol 2001; 69: 248-52.

[56] Tyner JW, Uchida O, Kajiwara N, et al. CCL5-CCR5 interaction provides antiapoptotic signals for macrophage survival during viral infection. Nat Med 2005; 11: 1180-7. https://doi.org/10.1038/nm1303

[57] Steinbach EC, Plevy SE. The role of macrophages and dendritic cells in the initiation of inflammation in IBD. Inflamm Bowel Dis 2014; 20: 166-75. https://doi.org/10.1097/MIB.0b013e3182a69dca

[58] Zhang $P$, Fan $Y$, Li Q, et al. Macrophage activating factor: A potential biomarker of periodontal health status. Arch Oral Biol 2016; 70: 94-9.

https://doi.org/10.1016/j.archoralbio.2016.06.010

[59] Katsiari CG, Liossis SNC, Sfikakis PP. The pathophysiologic role of monocytes and macrophages in systemic lupus erythematosus: a reappraisal. Semin Arthritis Rheum 2010; 39: 491-503. https://doi.org/10.1016/j.semarthrit.2008.11.002

[60] Zhang CY, Wei JF, He SH. Local base order influences the origin of ccr5 deletions mediated by DNA slip replication. Biochem Genet 2005; 43: 229-37.

https://doi.org/10.1007/s10528-005-5214-7

[61] Thomas JC. Characterization of the CCR5 chemokine receptor gene. Biochem Mol Biol Educ 2004; 32: 191-5. https://doi.org/10.1002/bmb.2004.494032030357

[62] Donyavi T, Bokharaei SF, Nahand JS, et al. Evaluation of CCR5- $\triangle 32$ Mutation among Individuals with High Risk Behaviours, Neonates Born to HIV-1 Infected Mothers, HIV-1 Infected Individuals, and Healthy People in an Iranian Population. J Med Virol 2020; 92: 1158-64. https://doi.org/10.1002/jmv.25658

[63] Estrada-Aguirre JA, Cázarez-Salazar SG, Ochoa-Ramírez LA, et al. Protective effect of CCR5 Delta-32 allele against HIV-1 in Mexican women. Curr HIV Res 2013; 11: 506-10. https://doi.org/10.2174/1570162X11666140101120225

[64] Trecarichi EM, Tumbarello M, Donati GK, et al. Partial protective effect of CCR5-Delta 32 heterozygosity in a cohort of heterosexual Italian HIV-1 exposed uninfected individuals. AIDS Res Ther 2006; 3: 22. https://doi.org/10.1186/1742-6405-3-22

[65] Abdolmohammadi R, Azar SS, Khosravi A, Shahbazi M CCR5 polymorphism as a protective factor for hepatocellular carcinoma in hepatitis B virus-infected Iranian patients. Asian Pac J Cancer Prev 2016; 17: 4643-6.

[66] Drozd DM, Gańczak M, Karpińska E. Concerns related to CCR5 gene delta 32 mutation role in hepatitis $B$ virus infection. Przegl Epidemiol 2017; 71: 571-81.

[67] Rodríguez-Rodríguez L, González-Juanatey C, GarcíaBermúdez $\mathrm{M}$, et al. CCR5 $\Delta 32$ variant and cardiovascular disease in patients with rheumatoid arthritis: a cohort study. Arthritis Res Ther 2011; 13: R133. https://doi.org/10.1186/ar3444

[68] Török N, Molnár K, Füvesi J, et al. Chemokine receptor $\mathrm{V}$ $\Delta 32$ deletion in multiple sclerosis patients in Csongrád County in Hungary and the North-Bácska region in Serbia. Hum Immunol 2015; 76: 59-64. https://doi.org/10.1016/j.humimm.2014.11.001

[69] Soto-Sánchez J, Santos-Juanes J, Coto-Segura $P$, et al. Genetic variation at the CCR5/CCR2 gene cluster and risk of psoriasis and psoriatic arthritis. Cytokine 2010; 50: 114-6. https://doi.org/10.1016/j.cyto.2010.01.006

[70] Von Hippel PT. The heterogeneity statistic $\mathrm{I}^{2}$ can be biased in small meta-analyses. BMC Med Res Methodol 2015; 15: 1-8. https://doi.org/10.1186/s12874-015-0024-z

[71] Rutter-Locher Z, Smith TO, Giles I, Sofat N. Association between systemic lupus erythematosus and periodontitis: a 
systematic review and meta-analysis. Front Immunol 2017; 8: 1295.

https://doi.org/10.3389/fimmu.2017.01295

[72] Pessoa L, Aleti G, Choudhury S, et al. Host-Oral Microbial Interactions in Lupus and Periodontal Diseases. Front Immunol 2019; 10: 2602 https://doi.org/10.3389/fimmu.2019.02602

[73] Samarani S, Dupont-Lucas C, Marcil V, et al. CpG Methylation in TGF $\beta 1$ and IL-6 Genes as Surrogate Biomarkers for Diagnosis of IBD in Children. Inflamm Bowel Dis 2020; 26: 1572-8. https://doi.org/10.1093/ibd/izaa074

[74] Silva, FRP Vasconcelos ACCG, França LFC, et al. Relationship between-889 $\mathrm{C} / \mathrm{T}$ polymorphism in interleukin$1 \mathrm{~A}$ gene and risk of chronic periodontitis: evidence from a meta-analysis with new published findings. Med Oral Patol Oral Cir Bucal 2017; 22: e7-e14.

[75] Silva FRP, Santos PL, Vasconcelos ACCG, et al. Polymorphisms in interleukins $17 \mathrm{~A}$ and $17 \mathrm{~F}$ genes and periodontitis: results from a meta-analysis. Mol Biol Rep 2017; 44: 443-53.

https://doi.org/10.1007/s11033-017-4128-x

[76] Silva FRP, Vasconcelos ACCG, França LFC, et al. Association between the rs1143634 polymorphism in interleukin-1B and chronic periodontitis: Results from a metaanalysis composed by 54 case/control studies. Gene 2018; 668: 97-106.

https://doi.org/10.1016/j.gene.2018.05.067
[77] Silva FRP, Galeno JG, Leal ALAB, et al. Non-significant association between- $330 \mathrm{~T} / \mathrm{G}$ polymorphism in interleukin-2 gene and chronic periodontitis: findings from a metaanalysis. BMC Oral Health 2020; 20: 58

https://doi.org/10.1186/s12903-020-1034-8

[78] Silva FRP, Leal ALAB, Nibali L, et al. Lack of association between mannose binding Lectin-2 gene polymorphisms and periodontitis: A meta-analysis. Meta Gene 2020; 100757. https://doi.org/10.1016/j.mgene.2020.100757

[79] Silva FRP, Pessoa LS, Shin JI, et al. Polymorphisms in the interleukin genes and chronic periodontitis: A field synopsis and revaluation by Bayesian approaches. Cytokine 2021; 138: 155361

https://doi.org/10.1016/j.cyto.2020.155361

[80] Ahmed HS, Ahmed H, Ad'hiah AH. Interleukin-1 single nucleotide polymorphisms and risk of systemic lupus erythematosus among Iraqi patients. Meta Gene 2020; 23: 100640.

https://doi.org/10.1016/j.mgene.2019.100640

[81] Nasiri M, Jaafari SM, Daryagard F, et al. Association of TIM3 (rs1036199) and TIM-4 (rs7700944, rs6882076) gene polymorphisms with susceptibility to systemic lupus erythematosus. Meta Gene 2020; 25: 100749. https://doi.org/10.1016/j.mgene.2020.100749

Received on 04-08-2021

Accepted on 19-09-2021

Published on 20-09-2021

https://doi.org/10.6000/1929-6029.2021.10.08

(C) 2021 da Silva et al.; Licensee Lifescience Global.

This is an open access article licensed under the terms of the Creative Commons Attribution License (http://creativecommons.org/licenses/by/4.0/) which permits unrestricted use, distribution and reproduction in any medium, provided the work is properly cited. 